\title{
Comparison of the Multi-Layer Multi-Configuration Time-Dependent Hartree (ML-MCTDH) Method and the Density Matrix Renormalization Group (DMRG) for Ground State Properties of Linear Rotor Chains
}

\author{
Samrit Mainali and Fabien Gatta) \\ Université Paris-Saclay, Institut des Sciences Moléculaires d'Orsay ISMO, UMR CNRS 8214 F-91405 Orsay, \\ France \\ Dmitri louchtchenko and Pierre-Nicholas Roy \\ Department of Chemistry, University of Waterloo, Waterloo, Ontario, N2L 3G1, Canada \\ Hans-Dieter Meyer (c) \\ Theoretische Chemie, Physikalisch-Chemisches Institut, Universität Heidelberg, Im Neuenheimer Feld 229, 69120 Heidelberg, \\ Germany
}

We demonstrate the applicability of the Multi-Layer Multi-Configuration Time-Dependent Hartree (ML-MCTDH) method to the problem of computing ground states of one-dimensional chains of linear rotors with dipolar interactions. Specifically, we successfully obtain energies, entanglement entropies, and orientational correlations that are in agreement with the Density Matrix Renormalization Group (DMRG), which has been previously used for this system. We find that the entropies calculated by ML-MCTDH for the larger system sizes contain a nonmonotonicity, as expected in the vicinity of a second-order quantum phase transition between ordered and disordered rotor states. We observe that this effect remains when all couplings besides nearest-neighbor are omitted from the Hamiltonian, which suggests that it is not sensitive to the rate of decay of the interactions. In contrast to DMRG, which is tailored to the one-dimensional case, ML-MCTDH (as implemented in the Heidelberg MCTDH package) requires more computational time and memory, although the requirements are still within reach of commodity hardware. The numerical convergence and computational demand of two practical implementations of ML-MCTDH and DMRG are presented in detail for various combinations of system parameters.

\section{INTRODUCTION}

With the possibility to control material processes at their most elementary level, it becomes even more necessary to develop new algorithms to numerically solve the Schrödinger equation 1 . In the field of molecular quantum dynamics, the Multi-Configuration Time-Dependent Hartree (MCTDH) approach $^{2 \cdot 6}$ has been applied to many different fields by various groups in the world: heterogeneous catalysis, reactive or non-reactive collisions, infrared spectroscopy, ultraviolet spectroscopy possibly involving non-Born-Oppenheimer processes, photochemistry, processes guided by laser pulses, optimal control, etc. MCTDH can be understood as a method that employs fully flexible time-dependent functions that follow the variational equations derived from the Dirac-Frenkel principle ${ }^{4}$. The method converges to the exact solution and can treat more Degrees Of Freedom (DOFs) than the quantum dynamics approaches relying on fixed time-independent functions. For instance, a standard benchmark case with MCTDH is the computation of the absorption spectrum of pyrazine taking into account all 24 DOFs and two excited electronic states $^{7}$. Another noteworthy application of $\mathrm{MCTDH}$ is the computation of the infrared (IR) absorption spectrum of the zundel cation $\frac{8}{10}, \mathrm{H}_{5} \mathrm{O}_{2}^{+}$, and its isotopomeres ${ }^{11}$. Finally, the MCTDH algorithm has been extended to solve the timeindependent Schrödinger equation to calculate eigenstates of a system with the so-called "improved relaxation" method ${ }^{14 \mid 15}$.

\footnotetext{
a)Electronic mail: fabien.gatti@ universite-paris-saclay.fr

b) Electronic mail: pnroy@uwaterloo.ca

c) Electronic mail: Hans-Dieter.Meyer@pci.uni-heidelberg.de
}

More recently, the Multi-Layer (ML) variant of $\mathrm{MCTDH}^{16}$ has been developed, which is able to treat quantum mechanically even higher-dimensional systems with more than 1000 DOFs22, 32. In ML-MCTDH, one makes a selection of layered effective modes through which MCTDH is applied in a recursive manner: the wavefunction is expressed in terms of time-dependent functions (first layer) that follow equations derived from the Dirac-Frenkel variational principle, but instead of expressing these functions in terms of timeindependent basis functions, they are themselves expressed in terms of lower-dimensional time-dependent functions (second layer) that also follow equations derived from the variational principle. The latter functions can themselves be expressed in terms of even lower time-dependent functions (next layer) or in terms of time-independent functions when we reach the last layer. In doing so, a very high flexibility is given to the MCTDH ansatz, and a very compact form of the wavefunction is obtained.

As it stands, the improved relaxation has already been combined with ML-MCTDH ${ }^{33 / 34}$, but has not yet been implemented in the Heidelberg package, which we use here. However, the ground state of a Hamiltonian can still be obtained by a propagation in imaginary time. To that end, we use the Heidelberg MCTDH package ${ }^{35}$, which contains an implementation of ML-MCTDH. The recursive tree structure of the ML-MCTDH ansatz can be viewed as a hierarchical Tucker decomposition, a kind of tensor decomposition (see Ref. 36 and references therein).

Another well-established tensor-based method is the Density Matrix Renormalization Group (DMRG), which is a numerical variational technique devised to study the low-energy physics of quantum many-body systems with high accuracy. Intro- 
duced by White in $1992^{37}$, DMRG has been proven to be particularly successful in condensed matter physics 3839 , quantum chemistry ${ }^{40,42}$, and molecular physics ${ }^{43}$. DMRG has been successfully applied to systems with more than one spatial dimension $^{44}$ and to real-time evolution ${ }^{45 / 47}$, but it is most efficient for computing ground states of long, one-dimensional systems. Note that the matrix product ansatz used in the DMRG method can be considered as a special case of the ansatz used in the ML-MCTDH method ${ }^{36}$. Despite the methods' shared tensor-based nature, there has been very little overlap between the communities working on ML-MCTDH and DMRG. A more systematic comparison of the two methods is appealing and could lead to important improvements for the simulations of molecular quantum systems ${ }^{36}$. The aim of the present paper is twofold: to compare the viability of these two methods for the calculation of rotational ground states; and to promote cross-pollination of the theory and implementation between ML-MCTDH and DMRG in the context of quantum molecular dynamics. We compare here the relative computational merits of ML-MCTDH and DMRG using two practical implementations of the two methods: we use two different program packages with different implementations. However, the comparison has a general scope. DMRG is an emerging method for quantum molecular dynamics (in particular the first application to chains of rotating dipolar molecules is very recen ${ }^{48}$ ), and the comparison to ML-MCTDH validates its relevance since MCTDH can be considered as the current standard for wavefunction based quantum molecular dynamics of large systems ${ }^{49}$.

In Ref. 48, DMRG was used to compute ground states of chains of rotors describing endofullerene "peapod" nanomolecular assemblies (NMAs), carbon nanotubes which contain fullerene cages with atoms or molecules trapped inside. By treating these nanomolecular assemblies as rigid 1D chains, it is possible to study the motion of the molecules enclosed inside. This approach has subsequently been applied to benchmark the Path Integral Ground State (PIGS) method for the computation of Rényi entanglement entropies in rotor chains using the replica trick ${ }^{50}$, and also to train neural network representations of the many-body states of interacting rotors using Restricted Boltzmann Machines (RBMs) ${ }^{\sqrt{51}}$.

In the following, we calculate three physical properties (energy, entanglement entropy, and orientational correlation) for systems of $N=10,25$, and 50 linear rotors with dipolar interactions using both ML-MCTDH and DMRG. Moreover, method-dependent properties (like memory usage and elapsed time) are compared between the two methods. The remainder of the paper is organized as follows: in section [II an overview of the two numerical methods is given, followed by the form of the Hamiltonian in section III] Section IV] presents the results, and we conclude with a brief summary in section $\mathrm{V}$

\section{THEORY}

We present in this section the essential aspects of the DMRG and ML-MCTDH methods used in our comparative study.

\section{A. DMRG}

The DMRG approach used in this work is described in detail in Ref. 48 In our implementation, the many-body wavefunction for a chain of quantum dipolar rotors has the form of a matrix product state (MPS) ansat $2 \frac{3839}{}$. We use the DMRG implementation from the ITensor package, where the Hamiltonian is treated as a matrix product operator (MPO) $)^{52}$. In a finite basis, the $N$-body wavefunction can be represented as a vector, $C_{\mathbf{n}}=\langle\mathbf{n} \mid \psi\rangle$, with multi-index $\mathbf{n}=\left(n_{1}, n_{2}, \ldots, n_{N}\right)$; see Fig. 11a for a schematic representation of a 10-dimensional tensor, corresponding to a wavefunction for 10 rotors. This vector can be written as an exact expansion in terms of products of matrices:

$$
\langle\mathbf{n} \mid \psi\rangle=\underline{\mathbf{A}}^{(1), n_{1}} \underline{\mathbf{A}}^{(2), n_{2}} \cdots \underline{\mathbf{A}}^{(N), n_{N}} .
$$

This MPS representation of $|\psi\rangle$ is formed from a set of matrices $\left\{\underline{\mathbf{A}}^{(k), n_{k}}\right\}$, where the index $n_{k}$ is associated with the physical site $k$, while the row and column indices of the matrices are referred to as bond indices. The so-called "bond dimension" is the common size between two adjacent matrices $\underline{\mathbf{A}}^{(k), n_{k}}$ and $\underline{\mathbf{A}}^{(k+1), n_{k+1}}$. Note that the first matrix, $\underline{\mathbf{A}}^{(1), n_{1}}$, acts as a row vector and the last one, $\underline{\mathbf{A}}^{(N), n_{N}}$, acts as a column vector; the end tensors $\underline{\mathbf{A}}^{(1)}$ and $\underline{\mathbf{A}}^{(\bar{N})}$ may be interpreted as either 2or 3-dimensional tensors, depending on the context. An MPS for 10 rotors is shown diagrammatically in Fig. 1 b. The final bond dimensions for the results presented below are given in the Supplementary Material.

The result of the matrix product is the scalar $\langle\mathbf{n} \mid \psi\rangle$, and the MPS expansion can be exact in principle. However, this is typically not desired, as it leads to the same exponential scaling one gets when using the full tensor. Instead, an approximation is introduced into the state by reducing the bond dimension. Although this reduction decreases the amount of entanglement that the state can contain, in practice one finds a balance between the level of approximation and the computational cost. In particular, certain states, such as area law states in one spatial dimension 53 , have entanglement that is independent of system size. For these states, the size of the MPS grows linearly with the number of sites, and DMRG is incredibly efficient.

A key aspect of the DMRG procedure is the Schmidt decomposition associated with a given partitioning of the system into parts $A$ and $B$,

$$
|\psi\rangle=\sum_{i} \sqrt{\lambda_{i}}\left|\xi_{i}^{A}\right\rangle \otimes\left|\xi_{i}^{B}\right\rangle,
$$

where each $\lambda_{i}$ is a non-negative real number, and $\left\{\left|\xi_{i}^{A}\right\rangle\right\}$ and $\left\{\left|\xi_{i}^{B}\right\rangle\right\}$ are orthonormal bases for $A$ and $B$. Numerically, these pieces may be obtained using the singular value decomposition (SVD), in which case the expansion coefficients are referred to as "singular values". An illustration of this decomposition is given in Fig.11, where $A$ and $B$ are shown to contain three and seven rotors, respectively. The DMRG procedure iteratively optimizes the MPS using a series of sweeps across every pair of adjacent sites, for which an effective Hamiltonian is diagonalized. Crucially, the result of each diagonalization is processed 
a)

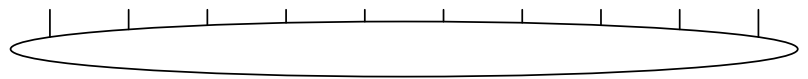

b)

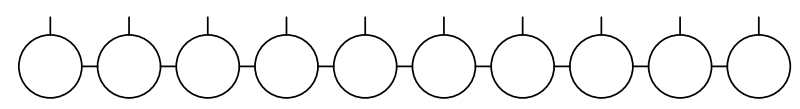

c)

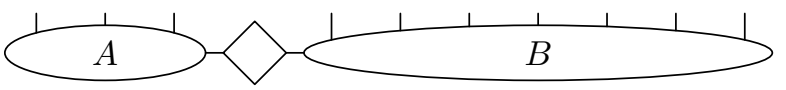

FIG. 1. Schematic depictions of tensor-based representations of a 10-rotor wavefunction. a) The full 10-dimensional tensor, $C_{\mathbf{n}}$, with vertical line segments corresponding to rotors. $b$ ) The matrix product state in Eq. (1), with circles representing the 3-dimensional tensors, $\underline{\mathbf{A}}^{(k)}$. Horizontal line segments imply tensor contraction along the bond indices (i.e. matrix multiplication). c) The Schmidt decomposition in Eq. (2), with the diamond denoting the diagonal matrix of singular values.

using the SVD by truncating singular values according to a cutoff criterion, such as

$$
\sum_{i} \lambda_{i} \leq \varepsilon
$$

where the sum runs over the truncated values. This truncation is vital to limiting the growth of the bond dimension. In the same way as an MPS, an MPO may also be compressed using the SVD, although improved approaches have been devised 4 4555.

\section{B. ML-MCTDH}

The wavepacket propagation calculations reported in this article are performed with the Heidelberg MCTDH package $e^{35}$. The MCTDH and ML-MCTDH methods are well documented $\sqrt{2+516 \sqrt[20]{20}}$ and are described here only briefly. Assuming that the system under consideration has $f$ degrees of freedom with coordinates $q_{1}, \ldots, q_{f}$, the ansatz for the MCTDH wavefunction reads:

$$
\Psi\left(q_{1}, \ldots, q_{f}, t\right)=\sum_{j_{1}=1}^{n_{1}} \cdots \sum_{j_{f}=1}^{n_{f}} A_{j_{1} \cdots j_{f}}(t) \prod_{\kappa=1}^{f} \varphi_{j_{\kappa}}^{(\kappa)}\left(q_{\kappa}, t\right) .
$$

Here $A_{j_{1} \cdots j_{f}}$ denotes the MCTDH expansion coefficients, and $\varphi^{(\kappa)}\left(q_{\kappa}, t\right)$ are the so-called single-particle functions (SPFs) for the degree of freedom $\kappa$. The SPFs are expressed in primitive basis sets or Discrete Variable Representation (DVR) grids:

$$
\varphi_{j_{\kappa}}^{(\kappa)}\left(q_{\kappa}, t\right)=\sum_{i_{\kappa}=1}^{N_{\kappa}} c_{i_{\kappa} j_{\kappa}}^{(\kappa)}(t) \chi_{i_{\kappa}}^{(\kappa)}\left(q_{\kappa}\right),
$$

where $\chi_{i_{\kappa}}^{(\kappa)}$ are orthonormal time-independent primitive basis functions of the $\kappa^{\text {th }}$ DOF. In our problem, $f=2 N$ with $N$ being the number of rotors (the number used to designate the $\mathrm{N}$-body wavefunction in DMRG) since there are two spherical angles to describe the rotation of each rotor.
The equations of motion for the A-coefficients and for the SPFs are derived ${ }^{2 \sqrt{5}}$ from the Dirac-Frenkel variational principle. These differential equations are non-linear and complicated, but the size of this set of coupled equations is in general much smaller than the set of equations obtained by expressing the wavefunction directly in a time-independent basis set.

Thus, the MCTDH method propagates a wavepacket on a small, time-dependent, variationally optimized basis set of single-particle functions, which in turn are defined on a timeindependent primitive basis sets with $N_{\kappa}$ functions for the $\kappa^{\text {th }}$ degree of freedom. In the limit $n_{\kappa} \rightarrow N_{\kappa}$, MCTDH becomes a numerically exact method to solve the Schrödinger equation within the primitive basis set. The SPFs are not restricted to be one-dimensional functions: they may depend on several coordinates, and in this case $q_{\kappa}$ is to be interpreted as a multidimensional variable and $f$ in Eq. (4) is to be replaced by the number of MCTDH particles, i.e. the number of combined modes.

The Multi-Layer (ML) variant ${ }^{16} \sqrt{20}$ of MCTDH provides a very efficient algorithm capable of treating quantum mechanically even higher-dimensional systems ${ }^{22 \mid 28}$. The key idea behind ML-MCTDH is to give more flexibility to the MCTDH ansatz by making an optimal choice of layered effective modes through which the MCTDH method is applied in a recursive manner. The ML approach enables one to represent the wavefunction in a very compact way. The particular structure of a ML-wavefunction, which has to be defined by the user, is given by a so called ML-tree. Through the ML-tree, one defines which modes of one layer are to be combined to build a mode of the layer above (see Fig. 2). Thus, the ML-MCTDH method uses trees, whereas DMRG conventionally uses an MPS. An MPS can be viewed as a very special tree, where the only flexibility within an MPS-tree is the ordering of the DOFs. An ML-tree, on the other hand, is very flexible 36156157 and its topology can be adjusted to the system. Note however that DMRG is not limited to MPSs $s^{36158}[60$.

\section{THE HAMILTONIAN}

Let us consider a system with $N$ identical rotors with rotational constant $B$ and dipole moment $\mu$, whose Hamiltonian reads:

$$
\hat{H}=\frac{B}{\hbar^{2}} \sum_{i=1}^{N} \hat{\ell}_{i}^{2}+\frac{\mu^{2}}{4 \pi \epsilon_{0}} \sum_{i=2}^{N} \sum_{j=1}^{i-1} \frac{\hat{V}_{i j}}{r_{i j}^{3}}
$$

where $r_{i j}$ is the distance between rotors $i$ and $j, \hat{V}_{i j}$ is the corresponding dipole-dipole potential operator, and $\hat{\ell}_{i}$ is the angular momentum operator of the $i^{\text {th }}$ rotor. Our goal is to describe a carbon nanotube peapod assembly, which is inherently linear. Thus, we may place the rotors along one axis, let's say the $z$ axis, and express the potential operator compactly as

$$
\hat{V}_{i j}^{(z)}=\hat{x}_{i} \hat{x}_{j}+\hat{y}_{i} \hat{y}_{j}-2 \hat{z}_{i} \hat{z}_{j},
$$

where $\left(x_{i}, y_{i}, z_{i}\right)$ denotes a unit vector pointing in the direction of the $i^{\text {th }}$ dipole. Due to the regular structure of a peapod NMA, 
we can position the rotors evenly with a lattice spacing, $r$, and write the nondimensionalized Hamiltonian as

$$
\frac{\hat{H}}{B}=\sum_{i=1}^{N} \frac{\hat{\ell}_{i}^{2}}{\hbar^{2}}+g \sum_{i=2}^{N} \sum_{j=1}^{i-1} \frac{\hat{V}_{i j}^{(z)}}{(i-j)^{3}},
$$

where the dimensionless parameter

$$
g=\frac{\mu^{2}}{4 \pi \epsilon_{0} r^{3} B}
$$

gives the strength of the dipole-dipole interaction.

As explained in Ref. 48 (see Figures 4 and 5 therein), this system appears to undergo a quantum phase transition when varying the parameter $g$ : two domains appear, corresponding to strongly and weakly interacting systems. The origin of this second-order phase transition between disordered and ordered phases has been suggested to arise from breaking of the rotational symmetry 61 .

As the above Hamiltonian contains all pairwise interactions, we refer to its realizations by the label "All". In addition, we consider the simplified Hamiltonian

$$
\frac{\hat{H}}{B}=\sum_{i=1}^{N} \frac{\hat{\ell}_{i}^{2}}{\hbar^{2}}+g \sum_{i=2}^{N} \hat{V}_{i, i-1}^{(z)},
$$

which retains only the nearest-neighbor couplings; its realizations are given the label "NN". Since it contains fewer terms, the NN Hamiltonian is computationally less taxing than the All Hamiltonian, but preserves the symmetries of the latter. Hence, we expect to see evidence of a quantum phase transition for the NN systems as well.

\section{RESULTS AND DISCUSSION}

We compare several quantities for the ground states of the systems defined by Eq. (8) and Eq. (10), as obtained by MLMCTDH and DMRG. In Ref.48, the dependence of the ground state energy on the number of rotors, $N$, obtained with DMRG is given in the form of chemical potentials; in the present paper we give the absolute energy values, $E$, for different choices of $N$ and $g$. We also compute the von Neumann entanglement entropy, defined as

$$
S_{\mathrm{vN}}=-\sum_{j} \lambda_{j} \ln \lambda_{j}
$$

where the $\lambda_{j}$ are the squares of the coefficients of the Schmidt decomposition in Eq. 2. For systems with an even number of rotors $(N=10$ and 50$)$, we use a symmetric splitting of the system into $A$ and $B$; for $N=25$, it is instead partitioned into 13 and 12 rotors.

As in Ref. 48, we have additionally calculated the expectation value of the "orientational correlation" (OC) operator:

$$
\frac{2}{N(N-1)} \sum_{i=2}^{N} \sum_{j=1}^{i-1} \hat{\mathbf{e}}_{i} \cdot \hat{\mathbf{e}}_{j},
$$

where the unit vector $\mathbf{e}_{i}=\left(x_{i}, y_{i}, z_{i}\right)$ describes the orientation of the $i^{\text {th }}$ rotor. Both the von Neumann entanglement entropy and the orientational correlation measure the correlations that are present in the system, but the former experiences a divergence with system size near $g=1.0$.

We first present highly converged ground state properties computed using DMRG (with the basis of spherical harmonics limited to $\ell \leq \ell_{\max }$ and making use of quantum number conservation ${ }^{48}$ ) in order to provide a benchmark for the comparison with ML-MCTDH. These results, presented in Tables I and II] have the highest level of convergence achieved in the present work, and were consequently quite computationally demanding to obtain. Note that in the case of only nearestneighbor couplings, the entanglement entropy still peaks at $g=1.5$ for the two larger system sizes, indicating that this phenomenon is likely driven by the symmetries of the Hamiltonian rather than its microscopic details, and providing further evidence of a quantum phase transition.

\section{A. Energies and orientational ordering}

The orientation of each rotor can be parameterized by two spherical coordinates: $\theta_{i}$ and $\phi_{i}$ for the $i^{\text {th }}$ rotor. For the ML-MCTDH calculations, we used a two-dimensional Extended Legendre ${ }^{62}$ Discrete Variable Representation (DVR) for each rotor (a DVR associated with spherical harmonics, called PLeg). With ML-MCTDH, several choices of layers are possible. In order to give the explicit ansatz of the MLMCTDH wavefunction, we display a graphical representation usually referred to as a tree. Fig. 2 depicts a 10-rotor chain described by a four-layer wavefunction. Each node (i.e. circle) represents a set of vectors of coefficients. A circle stands for a set of time-dependent expansion coefficients and a rectangle for a set of time-independent primitive basis functions (or DVR grids). The number next to each leg is the number of SPFs used in the calculation with $g=1.0$. Depending on the number of rotors and the value of $g$, the number of SPFs has been chosen to try to find a compromise between accuracy and Central Processing Unit (CPU) time for the calculations. At the top node we split the tree symmetrically into two halves to simplify the evaluation of the entanglement entropy: the $\lambda_{j}$ in Eq. (11) are given by the natural populations ${ }^{4}$ (i.e. eigenvalues of the reduced density matrix) of the top node. Then we mostly perform binary splittings, but in few cases a node has three children. We use 6 grid points for $\theta_{i}$ and 7 for $\phi_{i}$ (i.e. $\ell \leq 5$ and $m \leq 3$ ). We could have used smaller grid sizes for the low coupling cases, but in ML-MCTDH the computational effort depends only weakly on the grid sizes (although strongly on the numbers of SPFs), so that we decided to use identical grids throughout. Figures of trees for the 25- and 50-rotor problems as well as additional details on the ML-MCTDH and DMRG calculations are provided in the Supplementary Material.

In general, the dimensionality of the SPFs increases when one climbs up the tree and one expects that the numbers of SPFs needed for convergence should increase towards the top, but we see in Fig. 2 that this is not the case here. The reason is that another important factor is the effective coupling strength. 
TABLE I. DMRG parameters, results, and corresponding computational effort for systems with nearest-neighbor interactions (NN). Wall-times are given in hours "h", minutes "m", and seconds "s". Maximum memory usage and ground state MPS size are reported in MB.

\begin{tabular}{rcccclllrr}
\hline \hline$N$ & $g$ & $\ell_{\max }$ & \multicolumn{1}{c}{$\varepsilon$} & \multicolumn{1}{c}{$E$} & \multicolumn{1}{c}{$S_{\mathrm{vN}}$} & \multicolumn{1}{c}{ OC } & \multicolumn{1}{c}{ Time } & Memory & Size \\
\hline \multirow{4}{*}{10} & 0.5 & 5 & $10^{-14}$ & -0.3781091744 & 0.069105639 & 0.00533842 & $12 \mathrm{~m} 30 \mathrm{~s}$ & 54 & 1 \\
& 1.0 & 6 & $10^{-14}$ & -1.5597054109 & 0.2456055 & 0.03695576 & $42 \mathrm{~m} 59 \mathrm{~s}$ & 261 & 2 \\
& 1.5 & 7 & $10^{-14}$ & -3.7909948650 & 0.61805 & 0.184790 & $2 \mathrm{~h} 41 \mathrm{~m} 25 \mathrm{~s}$ & 1036 & 4 \\
& 2.0 & 8 & $10^{-14}$ & -7.383621680 & 0.7724429 & 0.369532 & $4 \mathrm{~h} 41 \mathrm{~m} 11 \mathrm{~s}$ & 1500 & 6 \\
& 0.5 & 5 & $10^{-14}$ & -1.009392863 & 0.06910577 & 0.0023572 & $39 \mathrm{~m} 18 \mathrm{~s}$ & 55 & 2 \\
25 & 1.0 & 6 & $10^{-14}$ & -4.184455427 & 0.24657055 & 0.0187613 & $3 \mathrm{~h} 14 \mathrm{~m} 17 \mathrm{~s}$ & 370 & 6 \\
& 1.5 & 7 & $10^{-14}$ & -10.491188621 & 0.786916 & 0.2467871 & $22 \mathrm{~h} 18 \mathrm{~m} 29 \mathrm{~s}$ & 2398 & 25 \\
& 2.0 & 8 & $10^{-14}$ & -20.775486204 & 0.77801316 & 0.41543072 & $23 \mathrm{~h} 15 \mathrm{~m} 03 \mathrm{~s}$ & 1861 & 23 \\
& 0.5 & 5 & $10^{-14}$ & -2.061532344 & 0.06910577 & 0.00121253 & $1 \mathrm{~h} 26 \mathrm{~m} 23 \mathrm{~s}$ & 57 & 3 \\
50 & 1.0 & 6 & $10^{-14}$ & -8.559041298 & 0.24657259 & 0.0100223 & $7 \mathrm{~h} 33 \mathrm{~m} 51 \mathrm{~s}$ & 431 & 14 \\
& 1.5 & 7 & $10^{-13}$ & -21.73364018 & 0.8213455 & 0.2788758 & $43 \mathrm{~h} 19 \mathrm{~m} 25 \mathrm{~s}$ & 2259 & 43 \\
& 2.0 & 8 & $10^{-14}$ & -43.103080319 & 0.778014411 & 0.430220817 & $60 \mathrm{~h} 58 \mathrm{~m} 57 \mathrm{~s}$ & 2059 & 52 \\
\hline \hline
\end{tabular}

TABLE II. DMRG parameters, results, and corresponding computational effort for systems with all pairwise interactions (All). Wall-times are given in hours "h", minutes "m", and seconds "s". Maximum memory usage and ground state MPS size are reported in MB.

\begin{tabular}{rcccclllrr}
\hline \hline$N$ & $g$ & $\ell_{\max }$ & \multicolumn{1}{c}{$\varepsilon$} & \multicolumn{1}{c}{$E$} & \multicolumn{1}{c}{$S_{\mathrm{vN}}$} & \multicolumn{1}{c}{ OC } & \multicolumn{1}{c}{ Time } & Memory & Size \\
\hline \multirow{4}{*}{10} & 0.5 & 5 & $10^{-14}$ & -0.398960623 & 0.079905618 & 0.00969951 & $24 \mathrm{~m} 35 \mathrm{~s}$ & 441 & 2 \\
& 1.0 & 7 & $10^{-14}$ & -1.800214523 & 0.3963543 & 0.09268385 & $3 \mathrm{~h} 40 \mathrm{~m} 56 \mathrm{~s}$ & 3958 & 9 \\
& 1.5 & 8 & $10^{-14}$ & -5.065659016 & 0.74355776 & 0.334380701 & $9 \mathrm{~h} 30 \mathrm{~m} 29 \mathrm{~s}$ & 9930 & 17 \\
& 2.0 & 8 & $10^{-14}$ & -9.869695663 & 0.744232934 & 0.447721340 & $9 \mathrm{~h} 28 \mathrm{~m} 06 \mathrm{~s}$ & 8838 & 16 \\
& 0.5 & 6 & $10^{-14}$ & -1.071398284 & 0.08016462 & 0.004763804 & $4 \mathrm{~h} 32 \mathrm{~m} 37 \mathrm{~s}$ & 1613 & 11 \\
25 & 1.0 & 7 & $10^{-14}$ & -5.036369456 & 0.5578798 & 0.1100315 & $58 \mathrm{~h} 48 \mathrm{~m} 22 \mathrm{~s}$ & 13271 & 80 \\
& 1.5 & 8 & $10^{-14}$ & -15.219922215 & 0.748988662 & 0.39127903 & $102 \mathrm{~h} 11 \mathrm{~m} 09 \mathrm{~s}$ & 17940 & 93 \\
& 2.0 & 8 & $10^{-14}$ & -29.04624326 & 0.741768134 & 0.48826618 & $76 \mathrm{~h} 55 \mathrm{~m} 59 \mathrm{~s}$ & 14742 & 75 \\
& 0.5 & 5 & $10^{-14}$ & -2.19213100 & 0.080169146 & 0.0025307 & $6 \mathrm{~h} 29 \mathrm{~m} 22 \mathrm{~s}$ & 1175 & 23 \\
50 & 1.0 & 7 & $10^{-13}$ & -10.48545272 & 0.712649 & 0.1319925 & $162 \mathrm{~h} 36 \mathrm{~m} 00 \mathrm{~s}$ & 16952 & 167 \\
& 1.5 & 7 & $10^{-14}$ & -32.2218434 & 0.74856170 & 0.409262540 & $158 \mathrm{~h} 38 \mathrm{~m} 18 \mathrm{~s}$ & 15255 & 204 \\
& 2.0 & 8 & $10^{-13}$ & -61.1002505 & 0.74150944 & 0.50184354 & $109 \mathrm{~h} 29 \mathrm{~m} 11 \mathrm{~s}$ & 8055 & 95 \\
\hline \hline
\end{tabular}

Here, the effective coupling strength decreases towards the ends of the chain, because at the ends, the rotors are less oriented than in the middle (this is why different groups have very different numbers of SPFs: for example, 20 versus 56 for the second layer in Fig. 2). At the top node, we always have comparatively few SPFs.

In order to have a comparable accuracy between MLMCTDH and DMRG, and to provide a meaningful comparison of the computational effort, the DMRG results are computed at a lower level of convergence than in Tables I and II for example, these DMRG calculations use $\ell_{\max } \leq 4$. The DMRG parameters were chosen to match the ML-MCTDH energies, and it can be seen in Table III that they are in close agreement. The difference is due to a small lack of convergence in MLMCTDH. Note that ML-MCTDH and DMRG simulations do not use the same primitive bases. As aforementioned, DMRG uses a basis set of spherical harmonics and ML-MCTDH uses a DVR based on spherical harmonics. They are thus different, but very similar. These DMRG calculations required between 9 and 25 primitive functions, making up a smaller local basis than the 42-point grid used by ML-MCTDH. The slower, but more converged benchmark DMRG calculations shown above included up to 81 basis functions.

The von Neumann entanglement entropies are given in Table IV and the expectation values of the orientational correlation operator are given in Table $\mathrm{V}$. That these values are nearly equal implies that both tensor decompositions are capable of faithfully representing these ground states. Following the DMRG results, the ML-MCTDH calculations quantitatively reproduce the conversion from ordered to disordered states: the von Neumann entropy and orientational correlation decrease to nearly zero with decreasing $g$, although the entropy occasionally does so nonmonotonically, as expected.

As always, we see that properties (like von Neumann entropy and orientational correlation) converge much slower than eigen-energies. Not surprisingly, the most difficult system for DMRG, with $N=50, g=1.0$, and all interactions (see Table II), is also very challenging for ML-MCTDH. Although the DMRG and ML-MCTDH energies differ by only a small fraction of a percent, the entanglement entropies and orienta- 


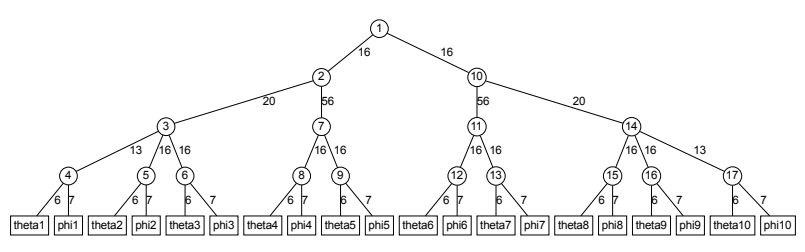

FIG. 2. Tree structure of the ML-MCTDH wavefunction for 10 rotors and $g=1.0$. As in Fig. 1. we have 10 rotors, but the rotation of each one is described by two angles $\theta$ and $\varphi$. In DMRG, as shown in Fig. 1 (c), the system of rotors is partitioned into two parts $A$ and $B$ and a procedure iteratively variationally optimizes the MPS using a series of sweeps across every pair of adjacent sites. In ML-MCTDH, the time-dependent SPFs are gathered in a tree: they depend on groups of coordinates that are fixed in the tree and are optimized variationally during the imaginary propagation.

tional correlations for this system disagree more strongly, by about $3 \%$ and $11 \%$.

\section{B. Computational effort}

Tables VI to VII outline the computational demands for the calculations performed with ML-MCTDH and DMRG. The ML-MCTDH calculations were done on a PC with an Intel I5-8500 CPU with 4 cores with a speed-up by about a factor of 3. The runs used all 4 cores via shared memory OpenMP parallelization, and the compiler was GCC 8.3.1 (gfortran). The three largest calculations, i.e. those with $N=50, g \geq 1.0$ and all pairwise interactions, would take several days on the PC, so they were run on a workstation. However, for better comparison, the wall-times of these calculations were rescaled to represent the 4-core PC wall-times. The DMRG code was built with the Intel MKL using GCC 8.3.0. For the ITensor library, we used the default -O2 setting for optimizations. Each calculation was run on a single Intel Xeon E5-2683 v4 core. For the sake of completeness, we have redone all the calculations with ML-MCTDH with the Intel Xeon E5-2683 v4 core for N = 10: the ML-MCTDH are roughly twice as slow. It shows that the choice of the computer is of importance in practice, but does not change the general trends observed below.

Table VI shows that we need greater wall-times to obtain ML-MCTDH results, and in fact much greater wall-times when all pairwise interactions are included (see the Supplementary Material for a brief discussion on this outcome). Note that no parallelization was used for the DMRG calculations. It appears that the ITensor implementation of DMRG is highly efficient for the present problem. Nevertheless, the present results show that ML-MCTDH can capture the main physics with reasonable wall-times. In particular, if the primary goal is to study the peak in the entanglement entropy, one may use the NN Hamiltonian, which requires wall-times that are more in line with those needed for DMRG.

The maximal memory usage data is presented in Table VII We observe that, with the exception of some small systems, the memory requirements of DMRG are systematically less than those of ML-MCTDH. In addition, the sizes of the final eigenstates are outlined in Table VIII, and we find that the MLMCTDH wavefunctions are not as compact as the equivalent MPSs. This is rather surprising since trees generally lead to more compact wavefunctions than MPS 36 56/57]. This probably shows that the one-dimensional rotor-chain problem fits very well to the DMRG algorithm.

TABLE III. Comparison of the (dimensionless) energies calculated with (a) ML-MCTDH and (b) DMRG for the ground state.

\begin{tabular}{cccccccc}
\hline \hline \multicolumn{5}{c}{$\mathrm{NN}$} \\
$g$ & $N=10$ & $N=25$ & $N=50$ & $N=10$ & $N=25$ & $N=50$ \\
\hline $0.5^{(a)}$ & -0.3781 & -1.0093 & -2.0611 & -0.3989 & -1.0713 & -2.1912 \\
$0.5^{(b)}$ & -0.3781 & -1.0093 & -2.0614 & -0.3989 & -1.0713 & -2.1919 \\
$1.0^{(a)}$ & -1.5597 & -4.1836 & -8.5573 & -1.8002 & -5.0351 & -10.480 \\
$1.0^{(b)}$ & -1.5597 & -4.1844 & -8.5590 & -1.8002 & -5.0362 & -10.485 \\
$1.5^{(a)}$ & -3.7908 & -10.490 & -21.726 & -5.0654 & -15.219 & -32.218 \\
$1.5^{(b)}$ & -3.7910 & -10.491 & -21.734 & -5.0656 & -15.220 & -32.222 \\
$2.0^{(a)}$ & -7.3836 & -20.774 & -43.095 & -9.8696 & -29.045 & -61.096 \\
$2.0^{(b)}$ & -7.3836 & -20.775 & -43.103 & -9.8695 & -29.046 & -61.099 \\
\hline \hline
\end{tabular}

TABLE IV. Comparison of the von Neumann entanglement entropies calculated with (a) ML-MCTDH and (b) DMRG for the ground state.

\begin{tabular}{ccccccc}
\hline \hline & \multicolumn{3}{c}{$\mathrm{NN}$} & \multicolumn{4}{c}{ All } \\
$g$ & $N=10$ & $N=25$ & $N=50$ & $N=10$ & $N=25$ & $N=50$ \\
\hline $0.5^{(a)}$ & 0.0691 & 0.0691 & 0.0688 & 0.0799 & 0.0800 & 0.0793 \\
$0.5^{(b)}$ & 0.0691 & 0.0691 & 0.0691 & 0.0799 & 0.0801 & 0.0801 \\
$1.0^{(a)}$ & 0.246 & 0.245 & 0.246 & 0.396 & 0.542 & 0.660 \\
$1.0^{(b)}$ & 0.246 & 0.247 & 0.247 & 0.396 & 0.558 & 0.712 \\
$1.5^{(a)}$ & 0.618 & 0.782 & 0.818 & 0.743 & 0.749 & 0.748 \\
$1.5^{(b)}$ & 0.618 & 0.787 & 0.821 & 0.744 & 0.749 & 0.749 \\
$2.0^{(a)}$ & 0.772 & 0.777 & 0.776 & 0.744 & 0.742 & 0.741 \\
$2.0^{(b)}$ & 0.772 & 0.778 & 0.778 & 0.744 & 0.742 & 0.742 \\
\hline \hline
\end{tabular}

TABLE V. Comparison of the orientational correlation (see Eq. 12p) calculated with (a) ML-MCTDH and (b) DMRG for the ground state.

\begin{tabular}{ccccccc}
\hline \hline \multicolumn{3}{c}{$\mathrm{NN}$} & \multicolumn{3}{c}{ All } \\
$g$ & $N=10$ & $N=25$ & $N=50$ & $N=10$ & $N=25$ & $N=50$ \\
\hline $0.5^{(a)}$ & 0.00534 & 0.00235 & 0.00121 & 0.009692 & 0.00477 & 0.00250 \\
$0.5^{(b)}$ & 0.00533 & 0.00235 & 0.00121 & 0.009689 & 0.00476 & 0.00253 \\
$1.0^{(a)}$ & 0.0369 & 0.0183 & 0.0100 & 0.0926 & 0.110 & 0.118 \\
$1.0^{(b)}$ & 0.0370 & 0.0188 & 0.0100 & 0.0927 & 0.110 & 0.132 \\
$1.5^{(a)}$ & 0.183 & 0.244 & 0.279 & 0.334 & 0.391 & 0.409 \\
$1.5^{(b)}$ & 0.185 & 0.247 & 0.279 & 0.334 & 0.391 & 0.409 \\
$2.0^{(a)}$ & 0.369 & 0.415 & 0.430 & 0.448 & 0.488 & 0.502 \\
$2.0^{(b)}$ & 0.370 & 0.415 & 0.430 & 0.448 & 0.488 & 0.502 \\
\hline \hline
\end{tabular}


TABLE VI. Comparison of the wall-times (in hours " $h$ ", minutes " $\mathrm{m}$ ", and seconds "s") needed to converge the ground state with (a) ML-MCTDH and (b) DMRG.

\begin{tabular}{ccccccc}
\hline \hline \multicolumn{3}{c}{$\mathrm{NN}$} & \multicolumn{4}{c}{ All } \\
$g$ & $N=10$ & $N=25$ & $N=50$ & $N=10$ & $N=25$ & $N=50$ \\
\hline $0.5^{(a)}$ & $0 \mathrm{~m} 32 \mathrm{~s}$ & $4 \mathrm{~m} 36 \mathrm{~s}$ & $7 \mathrm{~m} 25 \mathrm{~s}$ & $2 \mathrm{~m} 25 \mathrm{~s}$ & $1 \mathrm{~h} 11 \mathrm{~m}$ & $4 \mathrm{~h} 32 \mathrm{~m}$ \\
$0.5^{(b)}$ & $0 \mathrm{~m} 37 \mathrm{~s}$ & $1 \mathrm{~m} 44 \mathrm{~s}$ & $3 \mathrm{~m} 21 \mathrm{~s}$ & $0 \mathrm{~m} 39 \mathrm{~s}$ & $2 \mathrm{~m} 21 \mathrm{~s}$ & $4 \mathrm{~m} 41 \mathrm{~s}$ \\
$1.0^{(a)}$ & $2 \mathrm{~m} 06 \mathrm{~s}$ & $9 \mathrm{~m} 22 \mathrm{~s}$ & $1 \mathrm{~h} 09 \mathrm{~m}$ & $11 \mathrm{~m} 34 \mathrm{~s}$ & $5 \mathrm{~h} 00 \mathrm{~m}$ & $69 \mathrm{~h} 38 \mathrm{~m}$ \\
$1.0^{(b)}$ & $2 \mathrm{~m} 32 \mathrm{~s}$ & $7 \mathrm{~m} 11 \mathrm{~s}$ & $15 \mathrm{~m} 46 \mathrm{~s}$ & $3 \mathrm{~m} 09 \mathrm{~s}$ & $12 \mathrm{~m} 24 \mathrm{~s}$ & $40 \mathrm{~m} 52 \mathrm{~s}$ \\
$1.5^{(a)}$ & $2 \mathrm{~m} 48 \mathrm{~s}$ & $51 \mathrm{~m} 07 \mathrm{~s}$ & $2 \mathrm{~h} 08 \mathrm{~m}$ & $12 \mathrm{~m} 26 \mathrm{~s}$ & $12 \mathrm{~h} 09 \mathrm{~m}$ & $87 \mathrm{~h} 23 \mathrm{~m}$ \\
$1.5^{(b)}$ & $7 \mathrm{~m} 11 \mathrm{~s}$ & $25 \mathrm{~m} 22 \mathrm{~s}$ & $1 \mathrm{~h} 02 \mathrm{~m}$ & $9 \mathrm{~m} 27 \mathrm{~s}$ & $30 \mathrm{~m} 52 \mathrm{~s}$ & $1 \mathrm{~h} 07 \mathrm{~m}$ \\
$2.0^{(a)}$ & $11 \mathrm{~m} 19 \mathrm{~s}$ & $48 \mathrm{~m} 49 \mathrm{~s}$ & $1 \mathrm{~h} 56 \mathrm{~m}$ & $40 \mathrm{~m} 21 \mathrm{~s}$ & $12 \mathrm{~h} 49 \mathrm{~m}$ & $60 \mathrm{~h} 10 \mathrm{~m}$ \\
$2.0^{(b)}$ & $7 \mathrm{~m} 08 \mathrm{~s}$ & $25 \mathrm{~m} 09 \mathrm{~s}$ & $52 \mathrm{~m} 29 \mathrm{~s}$ & $8 \mathrm{~m} 57 \mathrm{~s}$ & $32 \mathrm{~m} 35 \mathrm{~s}$ & $1 \mathrm{~h} 09 \mathrm{~m}$ \\
\hline \hline
\end{tabular}

TABLE VII. Comparison of the maximum amount of memory (in $\mathrm{MB}$ ) required during the calculations with (a) ML-MCTDH and (b) DMRG.

\begin{tabular}{ccccccc}
\hline \hline & \multicolumn{3}{c}{$\mathrm{NN}$} & \multicolumn{4}{c}{ All } \\
$g$ & $N=10$ & $N=25$ & $N=50$ & $N=10$ & $N=25$ & $N=50$ \\
\hline $0.5^{(a)}$ & 29 & 94 & 149 & 30 & 100 & 198 \\
$0.5^{(b)}$ & 17 & 17 & 17 & 26 & 30 & 32 \\
$1.0^{(a)}$ & 64 & 142 & 448 & 65 & 165 & 555 \\
$1.0^{(b)}$ & 28 & 29 & 31 & 73 & 141 & 259 \\
$1.5^{(a)}$ & 64 & 275 & 536 & 65 & 281 & 595 \\
$1.5^{(b)}$ & 48 & 93 & 118 & 172 & 197 & 224 \\
$2.0^{(a)}$ & 145 & 278 & 548 & 147 & 292 & 619 \\
$2.0^{(b)}$ & 66 & 78 & 77 & 174 & 194 & 210 \\
\hline \hline
\end{tabular}

TABLE VIII. Comparison of the size (in MB) of the ground state wavefunction with (a) ML-MCTDH and (b) DMRG.

\begin{tabular}{ccccccc}
\hline \hline & \multicolumn{3}{c}{$\mathrm{NN}$} & \multicolumn{4}{c}{ All } \\
$g$ & $N=10$ & $N=25$ & $N=50$ & $N=10$ & $N=25$ & $N=50$ \\
\hline $0.5^{(a)}$ & 1.4 & 4.3 & 6.4 & 1.4 & 4.3 & 6.4 \\
$0.5^{(b)}$ & 0.03 & 0.1 & 0.2 & 0.07 & 0.3 & 0.6 \\
$1.0^{(a)}$ & 3.1 & 6.8 & 21.4 & 3.1 & 7.6 & 24.3 \\
$1.0^{(b)}$ & 0.1 & 0.4 & 0.9 & 0.3 & 2.1 & 7.2 \\
$1.5^{(a)}$ & 3.1 & 13.5 & 25.7 & 3.1 & 13.5 & 25.8 \\
$1.5^{(b)}$ & 0.4 & 1.8 & 4.9 & 0.7 & 3.0 & 7.0 \\
$2.0^{(a)}$ & 7.2 & 13.6 & 25.9 & 7.2 & 14.1 & 27.1 \\
$2.0^{(b)}$ & 0.4 & 1.4 & 3.2 & 0.7 & 2.4 & 5.3 \\
\hline \hline
\end{tabular}

\section{SUMMARY AND OUTLOOK}

We have performed a systematic comparison between two different numerical methods for calculating the ground states of a linear rotor model describing a nanomolecular assembly: the Multi-Layer (ML) variant of MCTDH and DMRG. The numerical performance and overall quality have been discussed for different values of the coupling strength and of the number of rotors involved in the chains. At present, the MLMCTDH method is both more memory- and time-consuming than DMRG, in some cases by more than one order of magnitude.

There are several factors working against ML-MCTDH for the dipolar rotor chain system. Firstly, ML-MCTDH is primarily a time-propagation method. As such, all variables are taken to be complex, whereas an eigensolver (such as the one used by DMRG) can be written using real arithmetic. Real arithmetic versus complex arithmetic inflates the memory demand by a factor of 2 and the computation time by almost a factor of 4 . Secondly, the relaxation method, i.e. propagation in imaginary time, is not a very efficient method to generate ground states, in particular when high accuracy is desired. Thirdly, as it is based on the MPS ansatz, DMRG is most efficient for computing grounds states of one-dimensional systems, as is the case here (as mentioned in the introduction DMRG can also be very efficient for excited states and time propagation). However, this efficiency may not generalize to higher dimensions. If one considered a 3D arrangement of rotors with e.g. 5 rotors in each direction, yielding 125 rotors altogether, then it is possible that ML-MCTDH will perform similarly as here, but DMRG could be more challenging to converge. As we point out in the Supplementary Material, several technical improvements are available, but they have yet to be implemented in the Heidelberg MCTDH software package which was used for the calculations in the present work. We hope that this contrast between the current capabilities of ML-MCTDH and DMRG motivates the development of these improvements.

Despite the difficulties faced by ML-MCTDH, it is able to capture the fundamental physics of the ground states of up to 50 rotors across a broad range of coupling strengths, which is per se an important result. This includes the entanglement entropy, whose nonmonotonic behaviour even in the NN case suggests the presence of a second-order quantum phase transition between ordered and disordered phases.

As explained by Larssor ${ }^{36}$, more systematic comparisons between ML-MCTDH and DMRG are highly desirable. Although the two methods have their own strengths, they are both based on tensor decompositions of the wavefunction, and the development of hybrid methods may be advantageous; for instance it could be profitable to use ML-MCTDH for timedependent simulations of a DMRG-optimized ground state. In addition, the combination of the experience of the two communities working on these two methods, and the techniques they have developed to solve the Schrödinger equation, may lead to a more efficient treatment of large quantum systems. In particular, more flexibility in the choice of the wavefunction representations for both methods could prove to be very helpful.

\section{SUPPLEMENTARY MATERIAL}

The supporting material provides the ML-trees for $N=$ 25 and 50 as well as some information about the integration scheme used for ML-MCTDH. It also presents a discussion about the Hamiltonian formats used for ML-MCTDH and 
DMRG and their impact on the efficiency of the two methods and how the form of the operator could be changed to improve the efficiency of ML-MCTDH. In addition, it gives some technical information about the DMRG calculations such as the size of the basis set, the truncation parameters and the final MPS bond dimensions.

\section{ACKNOWLEDGMENTS}

S.M. and F.G. acknowledge the use of computing center Mésolum of the LUMAT research federation (FR LUMAT 2764) and the help of its technical staff. D.I and P.-N.R. acknowledge the Natural Sciences and Engineering Research Council (NSERC) of Canada (RGPIN-2016-04403), the Ontario Ministry of Research and Innovation (MRI), the Canada Research Chair program (950-231024), the Canada Foundation for Innovation (CFI) (project No. 35232), the Canada First Research Excellence Fund (CFREF), the Shared Hierarchical Academic Research Computing Network (SHARCNET), and Compute Canada.

\section{DATA AVAILABILITY}

The data that support the findings of this study are available from the corresponding author upon reasonable request.

\section{REFERENCES}

${ }^{1}$ G. R. Fleming and M. A. Ratner. Grand challenges in basic energy sciences. Phys. Today 61 (2008), 28.

${ }^{2}$ H.-D. Meyer, U. Manthe, and L. S. Cederbaum. The multi-configurational time-dependent Hartree approach. Chem. Phys. Lett. 165 (1990), 73-78.

${ }^{3}$ U. Manthe, H.-D. Meyer, and L. S. Cederbaum. Wave-packet dynamics within the multiconfiguration Hartree framework: General aspects and application to NOCl. J. Chem. Phys. 97 (1992), 3199-3213.

${ }^{4}$ M. H. Beck, A. Jäckle, G. A. Worth, and H.-D. Meyer. The multiconfiguration time-dependent Hartree (MCTDH) method: A highly efficient algorithm for propagating wave packets. Phys. Rep 324 (2000), 1-105.

${ }^{5}$ H.-D. Meyer and G. A. Worth. Quantum molecular dynamics: Propagating wavepackets and density operators using the multiconfiguration time-dependent Hartree (MCTDH) method. Theor. Chem. Acc. 109 (2003), 251-267.

${ }^{6}$ H.-D. Meyer, F. Gatti, and G. A. Worth, Eds. Multidimensional Quantum Dynamics: MCTDH Theory and Applications. Wiley-VCH, Weinheim, 2009.

${ }^{7}$ A. Raab, G. Worth, H.-D. Meyer, and L. S. Cederbaum. Molecular dynamics of pyrazine after excitation to the $\mathrm{S}_{2}$ electronic state using a realistic 24mode model Hamiltonian. J. Chem. Phys. 110 (1999), 936-946.

${ }^{8} \mathrm{O}$. Vendrell, F. Gatti, and H.-D. Meyer. Dynamics and infrared spectroscopy of the protonated water dimer. Angew. Chem. Int. Ed. 46 (2007), 6918-6921.

${ }^{9}$ O. Vendrell, F. Gatti, D. Lauvergnat, and H.-D. Meyer. Full dimensional (15D) quantum-dynamical simulation of the protonated water dimer I: Hamiltonian setup and analysis of the ground vibrational state. J. Chem. Phys. 127 (2007), 184302.

${ }^{10}$ O. Vendrell, F. Gatti, and H.-D. Meyer. Full dimensional (15D) quantumdynamical simulation of the protonated water dimer II: Infrared spectrum and vibrational dynamics. J. Chem. Phys. 127 (2007), 184303.

${ }^{11} \mathrm{O}$. Vendrell, F. Gatti, and H.-D. Meyer. Strong isotope effects in the infrared spectrum of the zundel cation. Angew. Chem. Int. Ed. 48 (2009), 352 - 355.
${ }^{12}$ O. Vendrell, M. Brill, F. Gatti, D. Lauvergnat, and H.-D. Meyer. Full dimensional (15D) quantum-dynamical simulation of the protonated water dimer III: mixed Jacobi-valence parametrization and benchmark results for the zero-point energy, vibrationally excited states and infrared spectrum. J. Chem. Phys. 130 (2009), 234305.

${ }^{13}$ O. Vendrell, F. Gatti, and H.-D. Meyer. Full dimensional (15D) quantumdynamical simulation of the protonated water dimer IV: Isotope effects in the infrared spectra of $\mathrm{D}\left(\mathrm{D}_{2} \mathrm{O}\right)_{2}^{+}, \mathrm{H}\left(\mathrm{D}_{2} \mathrm{O}\right)_{2}^{+}$and $\mathrm{D}\left(\mathrm{H}_{2} \mathrm{O}\right)_{2}^{+}$isotopologues. J. Chem. Phys. 131 (2009), 034308.

${ }^{14}$ H.-D. Meyer, F. Le Quéré, C. Léonard, and F. Gatti. Calculation and selective population of vibrational levels with the Multiconfiguration TimeDependent Hartree (MCTDH) algorithm. Chem. Phys. 329 (2006), 179-192.

${ }^{15}$ L. J. Doriol, F. Gatti, C. Iung, and H.-D. Meyer. Computation of vibrational energy levels and eigenstates of fluoroform using the multiconfiguration time-dependent Hartree method. J. Chem. Phys. 129 (2008), 224109.

${ }^{16} \mathrm{H}$. Wang and M. Thoss. Multilayer formulation of the multiconfiguration time-dependent Hartree theory. J. Chem. Phys. 119 (2003), 1289-1299.

${ }^{17} \mathrm{U}$. Manthe. A multilayer multiconfigurational time-dependent Hartree approach for quantum dynamics on general potential energy surfaces. J. Chem. Phys. 128 (2008), 164116.

${ }^{18} \mathrm{H}$. Wang and M. Thoss. Numerically exact quantum dynamics for indistinguishable particles: The multilayer multiconfiguration time-dependent Hartree theory in second quantization representation. J. Chem. Phys. 131, 2 (2009), 024114.

${ }^{19}$ O. Vendrell and H.-D. Meyer. Multilayer multiconfiguration time-dependent Hartree method: Implementation and applications to a Henon-Heiles Hamiltonian and to pyrazine. J. Chem. Phys. 134 (2011), 044135.

${ }^{20} \mathrm{H}$. Wang. Multilayer Multiconfiguration Time-Dependent Hartree Theory. J. Phys. Chem. A 119 (2015), 7951.

${ }^{21} \mathrm{U}$. Manthe and T. Weike. On the multi-layer multi-configuration timedependent hartree approach for bosons and fermions. J. Chem. Phys. 146 (2017), 064117.

${ }^{22}$ H. Wang, D. E. Skinner, and M. Thoss. Calculation of reactive flux correlation functions for systems in a condensed phase environment: A multilayer multi-configuration time-dependent hartree approach. J. Chem. Phys. 125 (2006), 174502.

${ }^{23} \mathrm{H}$. Wang and M. Thoss. Quantum-mechanical evaluation of the Boltzmann operator in correlation functions for large molecular systems: A multilayer multiconfiguration time-dependent Hartree approach. J. Chem. Phys. 124 (2006), 034114.

${ }^{24} \mathrm{H}$. Wang and M. Thoss. Quantum dynamical simulation of electron-transfer reactions in an anharmonic environment. J. Phys. Chem. A 111 (2007), 10369.

${ }^{25}$ T. Westermann, R. Brodbeck, A. B. Rozhenko, W. Schoeller, and U. Manthe. Photodissociation of methyl iodide embedded in a host-guest complex: A full dimensional (189D) quantum dynamics study of $\mathrm{CH}_{3}$ I@ resorc[4]arene. J. Chem. Phys. 135 (2011), 184102.

${ }^{26} \mathrm{Q}$. Meng and H.-D. Meyer. A multilayer MCTDH study on the full dimensional vibronic dynamics of naphthalene and anthracene cations. J. Chem. Phys. 138 (2013), 014313.

${ }^{27}$ J. Schulze, M. F. Shibl, M. J. Al-Marri, and O. Kühn. Multi-Layer multiconfiguration time-dependent Hartree (ML-MCTDH) approach to the correlated exciton-vibrational dynamics in the FMO complex. J. Chem. Phys. 144 (2016), 185101.

${ }^{28}$ Y. Xie, J. Zheng, and Z. Lan. Full-dimensional multilayer multiconfigurational time-dependent Hartree study of electron transfer dynamics in the anthracene/C60 complex. J. Chem. Phys. 142 (2015), 084706.

${ }^{29}$ J. Zheng, Y. Xie, S. Jiang, and Z. Lan. Ultrafast nonadiabatic dynamics of singlet fission: Quantum dynamics with the Multilayer Multiconfigurational Time-Dependent Hartree (ML-MCTDH) method. J. Phys. Chem. C 120 (2016), 1375.

${ }^{30} \mathrm{Q}$. Meng and H.-D. Meyer. Lattice effects of surface cell: Multilayer multiconfiguration time-dependent Hartree study on surface scattering of CO/Cu(100). J. Chem. Phys. 146 (2017), 184305.

${ }^{31}$ D. Mendive-Tapia, T. Firmino, H.-D. Meyer, and F. Gatti. Towards a systematic convergence of Multi-Layer (ML) multi-configuration time-dependent Hartree nuclear wavefunctions: the ML-spawning algorithm. Chem. Phys. 482 (2017), 113-123.

${ }^{32}$ D. Mendive-Tapia, E. Mangaud, T. Firmino, A. de la Lande, M. DesouterLecomte, H.-D. Meyer, and F. Gatti. Multidimensional quantum mechan- 
ical modeling of electron transfer and electronic coherence in plant cryptochromes: The role of initial bath conditions. J. Phys. Chem. B 122 (2018), 126-136.

${ }^{33} \mathrm{H}$. Wang. Iterative calculation of energy eigenstates employing the multilayer multiconfiguration time-dependent Hartree theory. J. Phys. Chem. A 117 (2014), 9253.

${ }^{34} \mathrm{H}$. Wang and J. Shao. Quantum phase transition in the spin-boson model: A multilayer multiconfiguration time-dependent hartree study. J. Phys. Chem. A 123 (2019), 1882.

${ }^{35}$ G. A. Worth, M. H. Beck, A. Jäckle, O. Vendrell, and H.-D. Meyer. The MCTDH Package, Version 8.2, (2000). H.-D. Meyer, Version 8.3 (2002), Version 8.4 (2007). O. Vendrell and H.-D. Meyer Version 8.5 (2013). Version 8.5 contains the ML-MCTDH algorithm. Current versions: 8.4.20 and 8.5.13 (2020). See http://mctdh.uni-hd.de/ for a description of the Heidelberg MCTDH package.

${ }^{36} \mathrm{H}$. Larsson. Computing vibrational eigenstates with tree tensor network states (TTNS). J. Chem. Phys. 151 (2019), 204102.

${ }^{37} \mathrm{~S}$. White. Density matrix formulation for quantum renormalization groups. Phys. Rev. Lett. 69 (1992), 2863.

${ }^{38} \mathrm{U}$. Schollwöck. The density-matrix renormalization group. Rev. Mod. Phys. 77 (2005), 259.

${ }^{39} \mathrm{U}$. Schollwöck. The density-matrix renormalization group in the age of matrix product states. Ann. Phys. 326 (2011), 96.

${ }^{40} \mathrm{~S}$. R. White and R. L. Martin. Ab initio quantum chemistry using the density matrix renormalization group. J. Chem. Phys. 110, 9 (1999), 4127-4130.

${ }^{41}$ G. K.-L. Chan and S. Sharma. The density matrix renormalization group in quantum chemistry. Ann. Rev. Phys. Chem. 62 (2011), 465-481.

${ }^{42}$ C. Duperrouzel, P. Tecmer, K. Boguslawski, G. Barcza, Ö. Legeza, and P. W. Ayers. A quantum informational approach for dissecting chemical reactions. Chem. Phys. Lett. 621 (2015), 160-164.

${ }^{43}$ A. Baiardi and M. Reiher. The density matrix renormalization group in chemistry and molecular physics: Recent developments and new challenges. J. Chem. Phys. 152 (2020), 040903.

${ }^{44}$ E. M. Stoudenmire and S. R. White. Studying two-dimensional systems with the density matrix renormalization group. Annu. Rev. Condens. Matter Phys. 3 (2012), 111-128.

${ }^{45}$ J. Haegeman, C. Lubich, I. Oseledets, B. Vandereycken, and F. Verstraete. Unifying time evolution and optimization with matrix product states. Phys. Rev. B 94 (2016), 165116.

${ }^{46} \mathrm{Y}$. Kurashige. Matrix product state formulation of the multiconfiguration time-dependent Hartree theory. J. Chem. Phys. 149 (2018), 194114.

${ }^{47}$ S. Paeckel, T. Höhler, A. Swoboda, S. R. Mammana, U. Schollwöck, and C. Hubig. Time-evolution methods for matrix-product states. Ann. Phys. 411 (2019), 167998.

${ }^{48}$ D. Iouchtchenko and P.-N. Roy. Ground states of linear rotor chains via the density matrix renormalization group. J. Chem. Phys. 148 (2018), 134115.

${ }^{49}$ M. Motta, D. M. Ceperley, G. Kin-Lic Chan, J. A. Gomez, E. Gull, S. Guo, C. A. Jiménez-Hoyos, T. Nguyen Lan, J. Li, F. Ma, A. J. Millis, N. V. Prokofev, U. Ray, G. E. Scuseria, S. Sorella, E. M. Stoudenmire, Q. Sun, I. S. Tupitsyn, S. R. White, D. Zgid, and S. Zhang. Towards the solution of the many-electron problem in real materials: Equation of state of the hydrogen chain with state-of-the-art many-body methods. Phys. Rev. X 7 (2017), 031059.

${ }^{50}$ T. Sahoo, D. Iouchtchenko, C. Herdman, and P.-N. Roy. A path integral ground state replica trick approach for the computation of entanglement entropy of dipolar linear rotors. J. Chem. Phys. 152 (2020), 184113.

${ }^{51}$ I. J. S. De Vlugt, D. Iouchtchenko, E. Merali, P.-N. Roy, and R. G. Melko. Reconstructing quantum molecular rotor ground states. Phys. Rev. B $\mathbf{1 0 2}$ (2020), 035108.

${ }^{52}$ M. Fishman, S. R. White, and E. M. Stoudenmire. The ITensor software library for tensor network calculations. arXiv preprint (2020), arXiv:2007.14822.

${ }^{53}$ M. B. Hastings. An area law for one-dimensional quantum systems. J. Stat. Mech.: Theory Exp. 2007 (2007), P08024.

${ }^{54}$ D. E. Parker, X. Cao, and M. P. Zaletel. Local matrix product operators: Canonical form, compression, and control theory. Phys. Rev. B 102 (2020), 035147.

${ }^{55}$ G. Kin-Lic Chan, A. Keselman, N. Nakatani, Z. Li, and S. White. Matrix product operators, matrix product states, and ab initio density matrix renormalization group algorithms. J. Chem. Phys. 145 (2016), 014102.
${ }^{56}$ N. Nakatani and G. Kin-Lic Chan. Efficient tree tensor network states (ttns) for quantum chemistry: Generalizations of the density matrix renormalization group algorithm. J. Chem. Phys. 138 (2013), 134113.

${ }^{57} \mathrm{Z}$. Li. Expressibility of comb tensor network states (ctns) for the p-cluster and the femo-cofactor of nitrogenase. arXiv:2009.12573 (2020).

${ }^{58} \mathrm{R}$. Orús. A practical introduction to tensor networks: Matrix product states and projected entangled pair states. Annals of Physics 349 (2014), 117.

${ }^{59}$ V. Murg, F. Verstraete, $\mathscr{O}$. Legeza, and R. M. Noack. Simulating strongly correlated quantum systems with tree tensor networks. Phys. Rev. B 82 (2010), 205105.

${ }^{60}$ N. Chepiga and S. R. White. Comb tensor networks. Phys. Rev. B 99 (2019), 235426.

${ }^{61}$ B. Abolins, R. Zillich, and K. Whaley. A Ground State Monte Carlo Approach for Studies of Dipolar Systems with Rotational Degrees of Freedom. J. Low. Temp. Phys. 165 (2011), 249-260.

${ }^{62} \mathrm{~S}$. Sukiasyan and H.-D. Meyer. On the effect of initial rotation on reactivity. A multi-configuration time-dependent Hartree (MCTDH) wave-packet propagation study on the $\mathrm{H}+\mathrm{D}_{2}$ and $\mathrm{D}+\mathrm{H}_{2}$ reactive scattering systems. J. Phys. Chem. A 105 (2001), 2604-2611. 